http://jmscr.igmpublication.org/home/ ISSN (e)-2347-176x ISSN (p) 2455-0450

crossref DOI: https://dx.doi.org/10.18535/jmscr/v9i5.26

Journal Of Medical Science And Clinical Research

IGM Publication

An Official Publication of IGM Publication

\title{
Kirschner Wire Fixation for Mandible Fractures: A Simpler and Cost- Effective Technique
}

\author{
Authors \\ Dr Subrat Kumar Jena ${ }^{1}$, Dr Sourav Kumar Rout ${ }^{2}$ \\ ${ }^{1}$ Chief of Surgery and Head Department of Plastic, Hand and Microsurgery, Ashwini Hospital, Cuttack, \\ Odisha \\ ${ }^{2}$ Consultant Department of Cranio Maxillofacial Surgery, Ashwini Hospital, Cuttack, Odisha
}

\begin{abstract}
Introduction: In some centers across countries, Kirschner wires are still widely used for fixation of mandible fractures. Kirshner wire fixation for mandibular fracture can be a cost-effective alternative for fracture fixation. The present study is aimed at highlighting the relevance of Kirschner wire fixation a simple technique which gives desired result with much less cost and it is easier to learn.

Material and Method: A total of 254 patients with mandibular fractures treated by Kirschner wire fixation between March 2014 to February 2019 are prospectively analyzed. All patients complied to the uniform Kirschner wire care protocol, and maintained regular follow up for 6 months. Post-operative complication was categorized in two categories minor or major and further subdivided into pin tract infection, pin migration, pin loosening, delayed union, malunion, pin fracture, non-union, osteomyelitis, facial asymmetry and sensory nerve damage.

Result: A satisfactory treatment outcome was recorded in 221(87.4\%) of the 254 patients while complications occurred in 33(12.6\%) of the patients. Most complications were minor, commonly superficial pin tract infection in 10(6\%) patients. Major complication that led to revision surgery deep infection, malunion, nonunion. And fracture of pin. Development of infection is due to poor compliance with pin care.

Discussion: Kirschner Wire internal fixation can be a reasonable alternative for the surgical treatment of non-comminuted, and comminuted mandibular fractures and those without bone loss, in the setting of limited resources and minimally invasive technique.

Keywords: Mandibular fracture, Kirschner wire internal fixation, Minimally invasive, Cost-effective.
\end{abstract}

\section{Introduction}

Mandible fractures require accurate anatomic reduction and stable fixation because of their displacements and resulting functional and morphological consequences. This is at best achieved by open reduction and internal fixation. ${ }^{1}$ Titanium miniplates and screws technology is the gold standard in the modern treatment and widely used in developed countries while Kirschner wire fixation is an old but time honored and popular technique in resource limited circumstances. Especially, in the third world counties, where facial fractures comprise a significant proportion of trauma, paucity of resources precludes the use of plating technology and Kirschner wire osteosynthesis is still widely used..$^{2-3}$ Kirschner wire fixation can be an alternate method of fixation in cases of 
A) Unhealthy skin where implant fixation can lead to further infection.

B) Compound, comminuted devitalized bone fragments where implant placement is not indicated.

C) Certain institution which are not equipped with latest implant fixation system such as miniplates and micromotor drill set.

These are the relevant rationale for the present study.

However, there are very few data published on the results of Kirschner wire osteosynthesis for mandibular fractures from developing countries.

\section{Materials and Methods}

This is a prospective descriptive study carried out between March 2014 to February 2019. A total number of 254 patients were included in the study and Osteosynthesis was performed in all patients with mandibular fracture under general anesthesia. Consent for surgery and anesthesia was taken before the surgical procedures from all patients.

Inclusion criteria: Simple, compound, comminuted displaced or un-displaced mandible symphysis, parasymphysis and body, fractures with or without occlusion impairment.

Exclusion criteria: patient not fit for surgery under general anesthesia, edentulous patients; mandible fractures secondary to gunshot wounds; infected or defective mandible fractures; concomitant midfacial fractures; fractures with severe loss of overlying soft tissue necessitating local, regional, or free flap coverage; and condylar fractures. All the patients had clinical and radiographic evidence of fractures. (Figure 1)

The surgery was performed, usually within 0 to 10 days after the traumatic incident. Assessment of patient condition, pre-anestheticcheck-up was carried out to ensure fitness for surgery under general anesthesia.

In all the patient's extraoral approaches were used. The maxillomandibular fixation (MMF) using arch bars in patients with mandibular fractures was carried out. Following which a 2 mm-diameter stainless steel Kirschner wire was placed percutaneously using trocar and Calibri device to direct the Kirschner wire perpendicular to fracture segments Mandible symphysis, parasymphysis and body fracture was treated by two-point fixation to provide maximum rigidity after obtaining satisfactory reduction( figure 2). The reduction was checked by image intensifier through the procedure (figure 3). Kirschner wires left protruding through skin at the time of surgery (external, 220 pins) was covered with a dressing of petrolatum gauze and a soft bandage. We discharged 202 patients with a prescription for an oral antibiotic (1- to 3-day course) at the discretion of the treating surgeon, whereas 52 patients received no postoperative antibiotic prophylaxis.

Antibiotics were given preoperatively and continued for 7 to 10 days after surgery. A nonsteroidal anti-inflammatory drug was given for 3 days preoperatively and continued for 3 to 4 days postoperatively. Chlorhexidine mouth rinse was given when intraoral approach was used. Liquid and soft diet was recommended in patients with jaws immobilization. Patients were followed-up postoperatively for at least, 3, 6 weeks 3 months and 6 months and radiographic was taken for mandible osteosynthesis (figure 4). We documented and categorized pin-related problems into minor and major complication group (Table1).

Minor complications included redness, drainage, superficial pin track infection, pin loosening, pin migration, skin overgrowth, and delayed wound healing. A minor complication resolved with local wound care, early pin extraction, or both.

Major complications were defined as infections that required surgical drainage, septic arthritis, osteomyelitis, nonunion or malunionleading to additional surgery, and fracture through a pin track. Superficial pin track infection resolved with oral antibiotics, and pin track infection that did not resolve with local antibiotics necessitated incision and drainage in the operating room. Also, all the patients were checked for facial nerve impairment, operative site infection, and progress of bony 
union. At each visit, pin sites were evaluated, compliance with pin site care was recorded, and new radiographs were reviewed. In all cases, the data were recorded at the time of evaluation.

Fracture union was confirmed by clinical examination and radiographic evidence of callus formation and bridging trabeculae. Pins were removed at a mean of 6 weeks postoperatively. We documented and categorized pin-related problems into minor and major complication groups (table 1). Minor complications included redness, drainage. This study was reviewed and ethical committee clearance was granted.

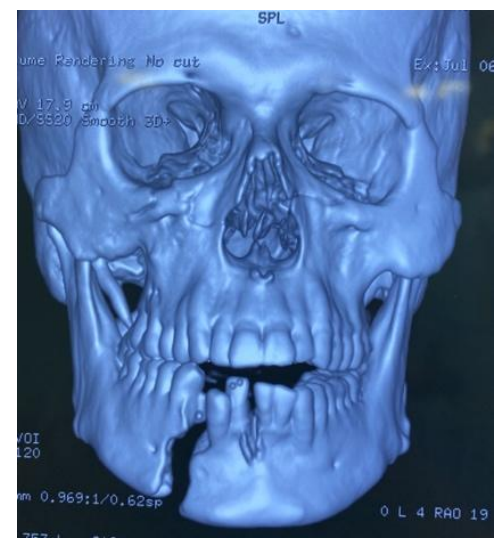

Figure 1

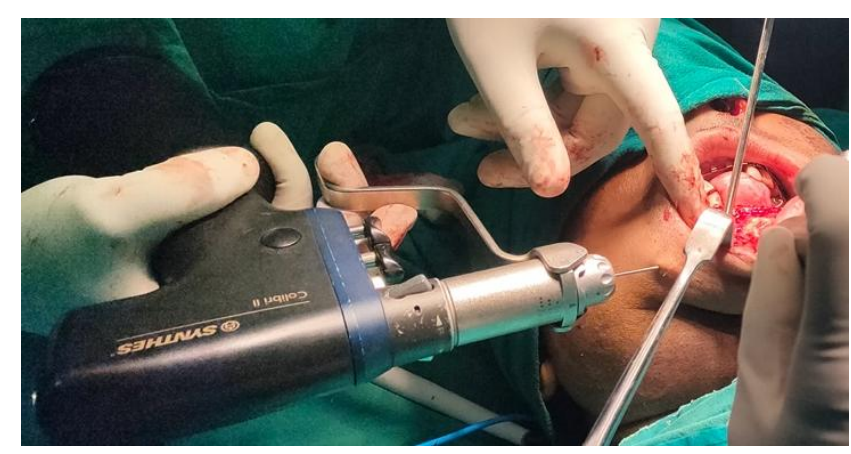

Figure 2

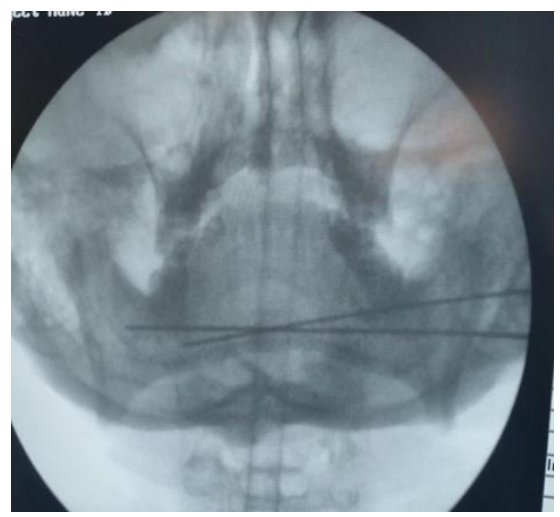

Figure 3

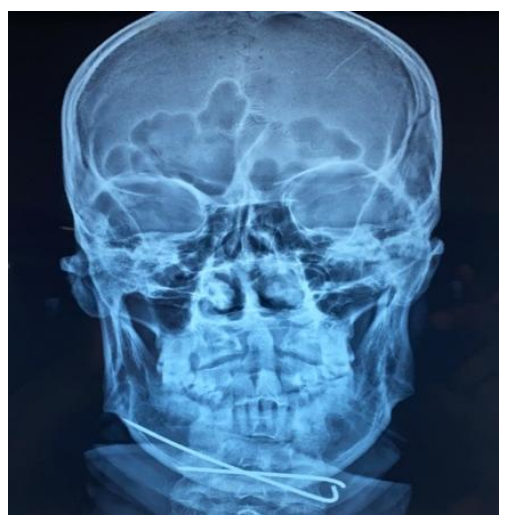

Figure 4

\section{Results}

Of the 254 patients, $(87.4 \%)$ had an uneventful and satisfactory treatment outcome as defined by a timely bone healing, restoration of the face morphology and the functions. Thirty-three patients $(12.6 \%)$ had some kind of complications. Operative site infection was the most common complication (Table 1). It consisted in surgical wound infection which resolved after systemic and local anti-microbial therapy. Sensory disturbance consisting in hypoesthesia or paresthesia of the lip, the chin, ipsilateral to the surgical side recovered in all the patients within 3 to 5 months postoperatively. Delayed bone union occurred in two patients who presented respectively a comminution and bone loss of the mandible.

The overall complications rate was $12.6 \%$ after mandibular osteosynthesis using Kirschner wire fixation. Total of 33 patients had Kirschner wire related complications. Minor complication occurred in 25(9.6\%) patients while major complication occurred in $8(3 \%)$ patients. Two patients had both minor and major complications.

Infectious complications occurred in 13 of 254 patients, Infections were diagnosed at an average of 18 days postoperatively. The vast majority of infections were minor, superficial pin track infections that resolved with a short course of oral antibiotics and local wound care. Only 1 patient in our study developed a deep abscess that necessitated incision, drainage, and removal of 2 Kirschner-wires. 
Kirschner wire fixation was carried out in symphysis region in $158(62.2 \%)$ patients, Para symphysis region in 50(19.6\%) patients, body region in $46(18.2 \%)$ patients. In these 24 patients had infection in the region of symphysis, 6 patients had infection in paraphysis and 3 patients in region of body (Table 2).

Table 1: Categories of K-Wire Complications

\begin{tabular}{|c|c|c|}
\hline Complication & cation type. & Patients(n) \\
\hline Total & & 33 \\
\hline Minor & & 25 \\
\hline Major & & 8 \\
\hline Total infectious & & 13 \\
\hline Redness & Minor & 2 \\
\hline Drainage & Minor & $\mathbf{1}$ \\
\hline $\begin{array}{l}\text { Superficial - } \\
\text { Pin infection }\end{array}$ & $\begin{array}{l}\text { Minor } \\
\text { Infectious }\end{array}$ & 10 \\
\hline Pin loosening & Minor & 3 \\
\hline Pin migration & Minor & 2 \\
\hline Skin overgrowth & Minor & $\mathbf{1}$ \\
\hline Delayed wound healing & Minor & 2 \\
\hline $\begin{array}{l}\text { Infection requiring- } \\
\text { Drainage }\end{array}$ & Major and infectious & 3 \\
\hline Nonunion requiring surger & y & 2 \\
\hline Malunion requiring surgery & y Major & 1 \\
\hline Fracture through pin track & Major & 2 \\
\hline Septic arthritis/osteomyeliti & tis Major and infectious & us 0 \\
\hline Malocclusion & Minor & 1 \\
\hline Sensory disturbances & Minor & 3 \\
\hline
\end{tabular}

Table 2: Kirschner wire location

\begin{tabular}{|lcc|}
\hline & & \\
\hline Pin location & Number of patients & Patients with Infection \\
\hline Symphysis & 158 & 24 \\
\hline Parasymphysis & 50 & 6 \\
\hline Body & 46 & 3 \\
\hline Total & 254 & 33 \\
\hline
\end{tabular}

\section{Discussion}

Fixation methods include intraosseous wiring, Kirschner wires, miniplates, resorbable implants, and lag screws. ${ }^{1,2}$ Selection of a method of fracture fixation depends on such factors as anatomic location of the fracture, timing of surgery, experience of the surgeon, age of the patient, and patient compliance. ${ }^{3,4}$ Miniplate fixation is believed to provide more functional stability than other methods but requires a secondary surgery for removal of the implant. The use of aresorbable miniplate obviates a second surgery, but resorbable miniplates have been shown to have less mechanical strength across a fracture site than nonresorbable miniplates. ${ }^{5}$

Open reduction and internal fixation of facial fracture can be technically challenging for unstable, displaced fractures and often requires additional extraoral incision for adequate visibility of fracture segments. Open reduction also requires stripping of periosteum for adequate reduction and fixation which compromises bone healing. ${ }^{3}$ In this study, more than $85 \%$ of the patients had a satisfactory outcome after Kirschner wire osteosynthesis without complication associated with open reduction and rigid internal fixation. 
Thus, internal/external Kirschner wire fixation ensures alignment and contact of the fracture fragments while maxilla-mandibular immobilization helps earlier osteosynthesis.

Kirchner wire osteosynthesis is an economical method of internal / external fixation. Kirschner wire and arch bars are more affordable and easily available than plates and screws. Unlike in the plate and screw system, there is no need of calibrated drill. Moreover, wire is a well-tolerated and non-cumbersome material which does not require removal if put internally. ${ }^{5}$ When used externally by percutaneous techniques, they are easily removed in the out-patient when radiological osteosynthesis is satisfactory. Moreover, the procedure is straightforward, and because the bone fragments can be immobilized precisely and firmly under fluoroscopic c arm guided vision. ${ }^{6}$ Insertion of the K-wire is not likely to comminute the segment.In this study, no patient complained of Kirshner wire intolerance as frequently encountered with plates. Some of disadvantages of Kirschner wire internal fixation, including percutaneous placement of wires cited in literatures is that Kirschner wires are not strong enough to prevent interfragmentary motion across the fractureand lack of directional control. ${ }^{6}$ However, we did not face this situation in our cases. Optimal use of image intensifier and good quality Kirschner wire drivers were helpful in seamless reduction and driving in of the Kirschner wires.

Maxillomandibular fixation was combined for additional stability during fracture union. Disadvantages of jaw fixation include aspiration, gingival trauma, difficulty with oral hygiene, delayed temporomandibular joint (TMJ) mobility, significant weight loss due difficulty of feeding, adverse effects on the patient's social and professional life due to speech difficulty. ${ }^{7}$ We did not face these problems by ensuring meticulous oral hygiene as well as maintaining strict and adequate calorie intake.

Lack of stability may result in delayed or nonunion as any mobility of the fracture fragments impedes the bony healing. However, well-placed wires and good case selection can avoid these complications. Kirschner wire fixation does away with the challenges of passing the wire through the drill holes in the fixation using Stainless steel wires. Especially in case of limited exposure of the fracture site and the wire break during its tightening are other concerns make Stainless steel wire internal fixation time consuming. ${ }^{8}$ Additionally, there is a risk of iatrogenic bone substance loss when passing the wire or during its tightening, particularly on the upper jaw bones or in case of comminuted fracture.

Postoperative complications ${ }^{9}$ of facial bone fracture include mal-occlusion, facial asymmetry, reduced mouth opening (trismus), impairment of vision, sensory disturbances zygomaticomaxillary fractures) and delayed bony union. Mal-occlusion may result from inadequate reduction and fixation of a Le Fort with or without a mandible fracture. However, a restored occlusion may be compromised by a noncompliant patient releasing prematurely himself the jaws immobilization. Delayed bone union observed in two patients with mandible fracture in this study can be due to lack of stability of the fixation as loss of substance or comminution of the fracture focus cannot be bridged effectively by Kirschner wire. Other complications include infections and osteomyelitis.

Dorr et al. ${ }^{10}$ detected a greater number of infections in patients who failed to routinely clean around protruding pins. However, the importance of scheduled pin site cleaning and the best method of pin care $^{11}$ remain debatable topics. Their spectrum varies from the surgical wound infection to osteomyelitis, differences of study designs and reporting bias. Although the operative site infection rates according to the fracture location do not reach significant differences in this study, even though in other studies mandible fractures are reported to be most common infectious complication amongst facial fractures.

Postoperative extended regimen prophylactic antibiotics $^{12,13}$ as routinely practiced in this study 
is reported to have no significant beneficial effect. Recognized limitations of our study include the accumulation of patient data at different time points, inconsistent use of antibiotics postoperatively, the lack of comparisons with other pin care regimens, the heterogeneity of the surgical procedures performed, and the short duration of follow-up observation.

Kim and Nam investigated the long-term clinical and radiological outcomes following the treatment of displaced or dislocated condylar fractures in children using threaded Kirschner wire and external rubber traction. The fractures were treated using threaded Kirschner wire and external rubber traction under portable $\mathrm{C}$-arm fluoroscopy. Their closed reduction technique in displaced or dislocated condylar fractures in children offered a reliable solution in preventing the unfavourable sequelae of closed treatment and the open technique, such as altered morphology, functional disturbances, and facial nerve damage. Similar results were obtained in our study in mandibular fractures fixation using non threaded Kirschner wire for body, para symphysis and symphysis fractures. $15,16,17$

\section{Conclusion}

Since the plating technology is not universally available and affordable in certain resource crunch situations, Kirschner wire internal fixation may be a reasonable alternative for the surgical treatment of simple, compound and comminuted mandibular fractures and those without bone loss. It is simple to learn and does not require elaborate instrumentation. They are better than Stainless Steel wire fixation (Inter-osseous wiring) and maxilla-mandibular fixation alone. However, efforts should be directed to adopt the modern technology of internal fixation using plating system because of many advantages including doing away with maxilla-mandibular immobilization.

\section{References}

1. Sugiura, T., Yamamoto, K., Murakami, K. and Sugimura, M. A comparative evaluation of osteosynthesis with lag screws, miniplates, or Kirschner wires for mandibular condylar process fractures. Journal of Oral and Maxillofacial Surgery 2001;59(10):1161-1168.

2. Sudheer R,Chakravarthy BD, Vura, N, Rajshekhar G. Management of Angle Mandible Fractures by 3D Rectangular Grid Plate: A Prospective Study. J. Maxillofac. Oral Surg.2020;19:420-424.

3. Gorzelnik, L. and Kozlovsky, E. BicorticalExtraoral Plating of Mandibular Fractures. Atlas of the Oral and Maxillofacial Surgery Clinics 2009; 17(1):35-43.

4. Richardson M, Hayes J, Jordan JR, Puckett A, \& Fort M. Biomechanical Evaluation of a Mandibular Spanning Plate Technique Compared to Standard Plating Techniques to Treat Mandibular Symphyseal Fractures. Surgery Research and Practice. 2015;2015:569030.

5. Lim HY, Jung CH, Kim SY, Cho JY, Ryu JY, Kim HM. Comparison of resorbable plates and titanium plates for fixation stability of combined mandibular symphysis and angle fractures. J Korean Assoc Oral Maxillofac Surg. 2014;40(6):285- 290.

6. Boutros SG. Closed reduction and fluoroscopically assisted percutaneous stabilization of displaced subcondylar mandible fractures. PlastReconstr Surg. 2005;116:971-977.

7. Sanam M, Kadakia S, Ducic Y. Postoperative Maxillomandibular Fixation After Open Reduction of Mandible Fractures. JAMA Facial Plast Surg. 2014;16(6):410-413.

8. Beogo R, Bouletreau P, Konsem T, Traore I, Coulibaly AT, Ouedraogo D. Wire internal fixation: an obsolete, yet valuable 
method for surgical management of facial fractures. Pan Afr Med J. 2014;17:219.

9. Morris, Lisa M. et al. Complications in Facial Trauma Facial Plastic Surgery Clinics, 2013; 21(4): 605 - 617.

10. Dorr MC, Backes M, Luitse, JSK, De Jong, V M \&Schepers T. Complications of Kirschner Wire Use in Open Reduction and Internal Fixation of Calcaneal Fractures. The Journal of Foot and Ankle Surgery.2016; 55(5): 915-917.

Lethaby A, Temple J, Santy J. Pin site care for preventing infections associated with external bone fixators and pins. Cochrane Database Syst Rev 2008;4:CD004551.

11. Zosa, BM, Elliott CW, Kurlander DE, Johnson F, Ho VP, \& Claridge JA. Facing the facts on prophylactic antibiotics for facial fractures. Journal of Trauma and Acute Care Surgery.2018; 85(3): 444-450.

12. Odom EB, \& Snyder WAK. Mandible Fracture Complications and Infection. Plastic and Reconstructive Surgery.2016; 138(2):282e-289e.

13. Haghighi K, Manolakakis MG, Balog C. Open Reduction with K-Wire Stabilization of Fracture Dislocations of the Mandibular Condyle: A Retrospective Review. J Oral Maxillofac Surg. 2017;75(6):1238.e11238.e7.

14. Kim JH, Nam DH. Closed reduction of displaced or dislocated mandibular condyle fractures in children using threaded Kirschner wire and external rubber traction. Int J Oral Maxillofac Surg. 2015;44(10):1255-1259.

15. Haghighi, K, ManolakakisMG, \& Balog C. Open Reduction With K-Wire Stabilization of Fracture Dislocations of the Mandibular Condyle: A Retrospective Review. Journal of Oral and Maxillofacial Surgery, 2017;75(6): 1238.e1-1238.e7.
16. Kang DH, Jung DW, Yong HK, Kim TG, Lee JH, Chung KJ. Kirschner wire fixation for the treatment of comminuted zygomatic fractures. Archives of craniofacial surgery 2015;16(3):119-124. 